\title{
MathTOUCH: Mathematical Input Interface for E-Assessment Systems
}

Shizuka Shirai, Department of Human Environmental Sciences, Mukogawa Women's University, Nishinomiya, Japan. Email: shirai@mukogawa-u.ac.jp

Tetsuo Fukui, Department of Human Environmental Sciences, Mukogawa Women's University, Nishinomiya, Japan. Email: fukui@mukogawa-u.ac.jp

\begin{abstract}
In 2012, we developed a new mathematical input interface with Java, named MathTOUCH. The interface facilitates the acceptance of a mathematical expression as input by interactively converting from a colloquial-style mathematical text (string). This input method enables users to input almost any mathematical expression without learning a new language or syntax. However, the user requires a Java-compliant device for mathematical input. In this study, we developed a reconstructed version of MathTOUCH by using JavaScript (HTML5) to enable the use of MathTOUCH across various devices. The result of our evaluation showed that students are able to practice their mathematical work on STACK using the reconstructed MathTOUCH as well as the previous version of MathTOUCH.
\end{abstract}

Keywords: math input interface, mathematics, human-computer interaction, STACK.

\section{Introduction}

In recent years, e-assessment systems, for example STACK, WeBWorK, Maple T.A., Numbas, and Math on Web (Osaka Prefecture University, 2004), whereby learners are able to answer mathematical questions by entering mathematical expressions, have been used for instructing students at many universities. However, current standard interfaces that accept mathematical expressions as input are either text-based or structure-based interfaces, and are cumbersome for novice learners to use. For example, text-based interfaces accept input according to the CAS command syntax. Furthermore, it is difficult for the users to imagine the desired mathematical expressions because the input is not in WYSIWYG format. On the other hand, the advantage of structure-based interfaces is that learners are able to operate in WYSIWYG. In addition, users are able to input mathematical template icons with the help of a GUI. Therefore, they do not need to remember CAS command syntax as for the text-based interface. However, users have to understand the structure of the mathematical expressions they require and should be able to select the mathematical template icons from the GUI in the correct order (Pollanen, Wisniewski and Yu, 2007). Furthermore, it is troublesome to make corrections later (Smithies, Novins and Arvo, 2001).

Fukui (2012) attempted to overcome these shortcomings by proposing a new mathematical input interface, named MathTOUCH. MathTOUCH facilitates interactive conversion from a colloquial-style mathematical text to the desired two-dimensional mathematical expressions. The results of a previous study of ours (e.g. Shirai and Fukui, 2014) showed that novice mathematics learners found MathTOUCH to be user-friendly. However, the users have to use a Java-compliant device in order to use MathTOUCH.

In this study, we reconstructed MathTOUCH using JavaScript to make MathTOUCH available not only on Java-compliant devices but also on various other devices. We evaluated the effectiveness of the reconstructed version of MathTOUCH (hereafter abbreviated as RMT) by investigating whether students are able to practice mathematical work using the reconstructed MathTOUCH at the same learning rate as with MathTOUCH based on Java (hereafter abbreviated as PMT). 


\section{Proposed interface}

\subsection{MathTOUCH}

MathTOUCH is a mathematical input interface developed using Java. MathTOUCH enables users to input the desired mathematical expressions by converting colloquial-style linear strings (Fukui, 2012). For example, if users would like to enter $\frac{1}{a^{2}+3}$, they only have to enter " $1 / \mathrm{a} 2+3$ " (see step 1 of figure 1). Neither do they need to input a power sign (e.g. a caret symbol) nor parentheses for the delimiters. In other words, users do not need to enter symbols that are not printed. Next, users perform the conversion to the desired two-dimensional mathematical expressions on an element starting from the left. They have only to select interactively the desired elements and/or the operands from the candidates by using MathTOUCH (see step 2 of figure 1). Finally, after they fixed all the elements, they are able to obtain the mathematical expressions in the desired format. The available output formats are LaTeX, MathML, PNG, JPEG, EPS, Maxima, Maple, and Mathematica. Table 1 presents examples of mathematical expressions and corresponding linear strings that can be entered into MathTOUCH. This input method enables users to input almost all mathematical expressions without learning a new language or syntax.

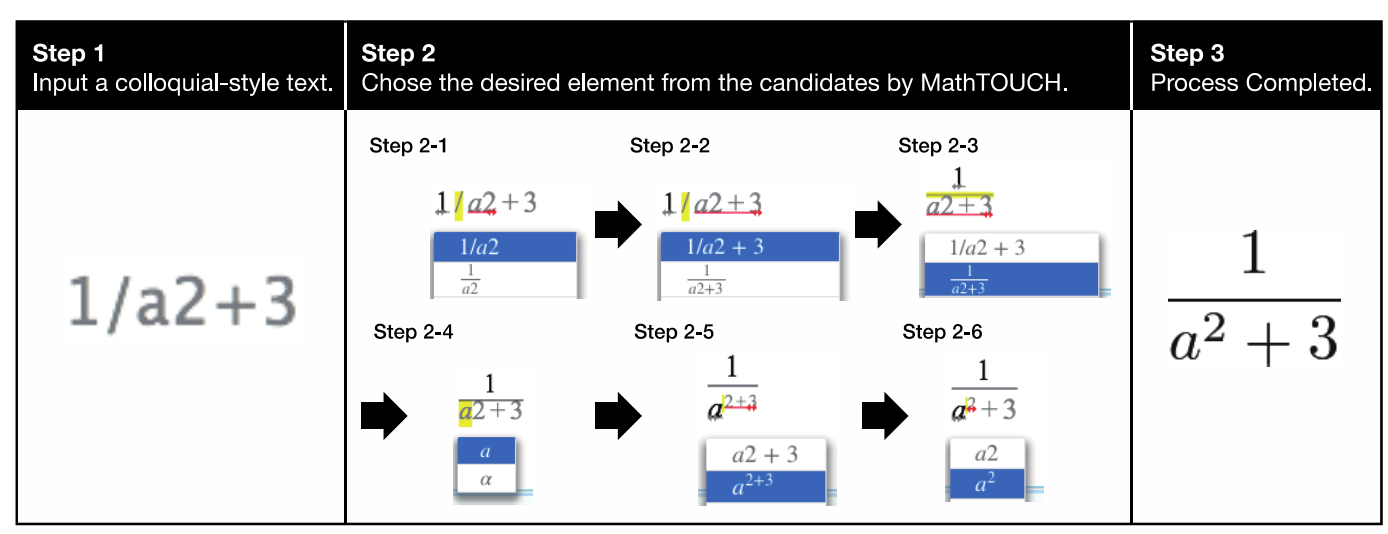

Figure 1. MathTOUCH input procedure.

Table 1. Example mathematical expressions using MathTOUCH.

\begin{tabular}{c|c}
\hline \hline Example & MathTOUCH \\
\hline $5 x^{2}+2$ & $5 \times 2+2$ \\
$\sqrt{2}$ & $\operatorname{root2}$ \\
$\sin ^{2} x$ & $\sin 2 \mathrm{x}$ \\
$\log _{10} x$ & $\log 10 \mathrm{x}$ \\
$e^{\pi x}$ & epx \\
$\sum_{k=1}^{n} k^{2}$ & sumk=1nk2 \\
$\lim _{x \rightarrow 1} \frac{x}{2}$ & $\operatorname{limx}-->1 \mathrm{x} / 2$ \\
$\frac{d f}{d x}$ & $\mathrm{df} / \mathrm{dx}$ \\
$\int_{0}^{1} x(1-x) d x$ & $\operatorname{int} 01 \mathrm{x}(1-\mathrm{x}) \mathrm{dx}$ \\
\hline
\end{tabular}




\subsection{Advantages of MathTOUCH}

In our previous study, we conducted two experiments, i.e. a performance survey (Shirai, Nakamura and Fukui, 2015) and an eight-week learning experiment (Shirai and Fukui, 2014), to evaluate the efficacy of MathTOUCH. In this section, we summarise these studies.

The performance survey focused on the following research question: Are students able to input mathematical expressions using MathTOUCH more smoothly than with the standard interfaces found in current e-assessment systems? In this study, we carried out a mathematical entering test to compare MathTOUCH, a text-based interface, and a structure-based interface regarding three elements of usability: effectiveness, efficiency, and satisfaction. The number of participants was 108 including 54 high school students and 54 university students. The ratio of humanities courses to science courses and males to females among participants was almost the same. They were asked to enter mathematical expressions using one of the three interfaces assigned to them. The results of a Mann-Whitney test showed no significant difference for the task-performance rates among the three interfaces. Regarding the task-performance times, MathTOUCH enabled participants to enter mathematical expressions approximately 1.2 to 1.6 times faster than the standard interfaces. Moreover, our system was shown to have a high level of user satisfaction in regards to mathematics input usability.

The eight-week learning experiment focused on the following research question: are students able to practice mathematical work using MathTOUCH on STACK at the same learning rate as with the current interface on STACK? In this intersubject study, 84 students, who showed no significant difference regarding mathematical skill based on a previous paper test, practiced mathematical work on STACK using their assigned mathematical input interface for eight weeks. The results showed that students were able to practice using MathTOUCH at the same learning rate. Furthermore, the results of the questionnaire revealed a higher level of satisfaction regarding Memorability, which was significantly higher than with the current interface.

These above results indicate that MathTOUCH is more effective in terms of input performance than current standard interfaces. However, users needed to use a Java-compliant device in order to interact with MathTOUCH.

\subsection{Reconstructed MathTOUCH}

In this section, we describe how we reconstructed MathTOUCH such that the interface is not only available on Java-compliant devices but also on various other devices. We reconstructed MathTOUCH using JavaScript (HTML5) because JavaScript is compatible with web applications. Developers are able to incorporate MathTOUCH into their own web applications and only have to include information of the header and body for MathTOUCH. Figure 2 shows a screenshot of the interface of the new version of MathTOUCH implemented on STACK. We used MathJax to display the conversion candidates. Furthermore, we added an edit function and enhanced the support function. The procedure for entering expressions is the same as with MathTOUCH using Java. 


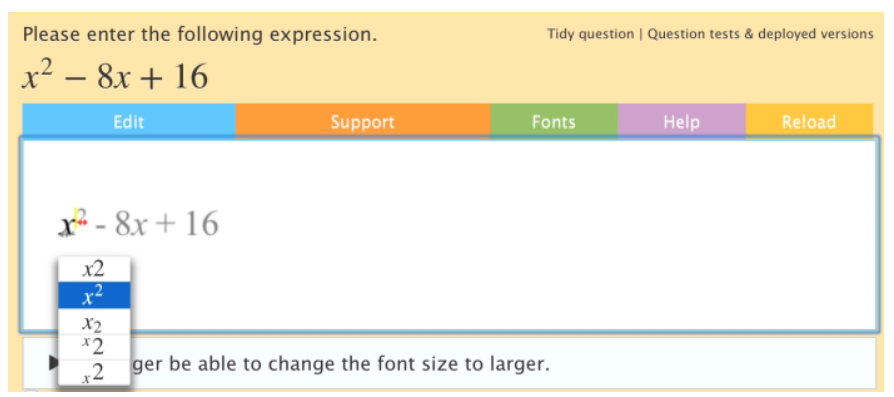

Figure 2. Reconstructed MathTOUCH on STACK.

\section{Evaluation}

\subsection{Purpose and Procedure}

Our prior work showed that the input performance of MathTOUCH exceeds that of current standard interfaces. We conducted a five-week learning experiment to test whether the RMT version offered stable input performance in comparison to the PMT version. The experiments are intended to investigate whether students are able to practice mathematical work using RMT on STACK at the same learning rate as PMT.

This experimental study involved 30 students as participants who were assigned to two groups. One group was assigned RMT ( $N=16)$, whereas the other group was assigned PMT $(\mathrm{N}=14)$. We conducted a pre-survey to investigate whether any differences in terms of typing skill and basic mathematical knowledge existed between the groups. The results showed that the difference between the groups was not significant. Every week for 5 weeks, they practiced mathematical work on STACK using their assigned mathematical input interface. Table 2 contains examples of the questions. The mathematical content involved basic calculations with square roots. They practiced 10 questions once a week.

\subsection{Measures}

We measured the solving times, the percentage of correct answers, and the learning rates. Moodle automatically measures the total amount of time spent on quizzes from the moment the button is clicked to start the quiz until the button is clicked to submit all the answers. We took the mean time for each group to be the solving time. After they completed all the mathematical work, we administered a questionnaire to determine students' subjective satisfaction with each interface. We used a 5-point rating scale from 1 (strongly disagree) to 5 (strongly agree). The contents of the questionnaire are shown in the first column of table 3.

Table 2. Mathematical example of mathematics drill questions.

\begin{tabular}{c|c}
\hline \hline Example of questions & Answer \\
\hline Simplify the expressions. $\sqrt{20} \times 2 \sqrt{2} \div \sqrt{5}$ & $4 \sqrt{2}$ \\
Simplify the expressions. $\sqrt{35} \times \sqrt{5}-\frac{14}{\sqrt{7}}$ & $3 \sqrt{7}$ \\
\hline
\end{tabular}


Table 3. Results of the questionnaire regarding subjective satisfaction.

\begin{tabular}{|c|c|c|}
\hline Contents of the questionnaire & PMT & RMT \\
\hline It was easy to master the use of this UI. & $3.94(0.85)$ & $3.86(1.03)$ \\
\hline Mathematical expressions could be inputted smoothly using this UI. Efficiency & $3.81(0.91)$ & $3.71(0.99)$ \\
\hline It was easy for me to correct miss-entered operations. & $3.63(0.89)$ & $3.14(0.86)$ \\
\hline $\begin{array}{l}\text { Even after the second week, I remembered how to use this UI I was instructed } \\
\text { on in the first week. }\end{array}$ & $4.31(0.70)$ & $3.79(1.19)$ \\
\hline $\begin{array}{c}\text { Would you like to use this UI when you enter the mathematical expressions? } \\
\text { Loyalty }\end{array}$ & $3.88(0.62)$ & $3.64(1.34)$ \\
\hline
\end{tabular}

Numbers in parentheses denote SD.

\subsection{Results}

Figure 3 shows the result of the solving time and percentage of correct answers. We ran a MannWhitney test for each week. This analysis yielded no significant interaction between PMT and RMT for each week.

We also calculated the learning rate using the log-linear model by progressive average. We excluded the data for the first week because the students had been given instruction on how to use the system on that same day. The five-week coefficient of determination $\left(R^{2}\right)$ for PMT and RMT is 0.910 and 0.801 , respectively. On the other hand, when the first week is excluded, the four-week $R^{2}$ for PMT and RMT is 0.998 and 0.991 , respectively. These results support the validity of use for the 4 weeks of data for evaluating learning rate. The results of the learning rate show that students are able to practice mathematical work using RMT with the same learning rate as with PMT with $92.4 \%$ and $89.9 \%$, respectively.

Table 3 provides the results for the subjective satisfaction. The results of the Mann-Whitney test showed no significant difference for the solving times between PMT and RMT for each of the questions.

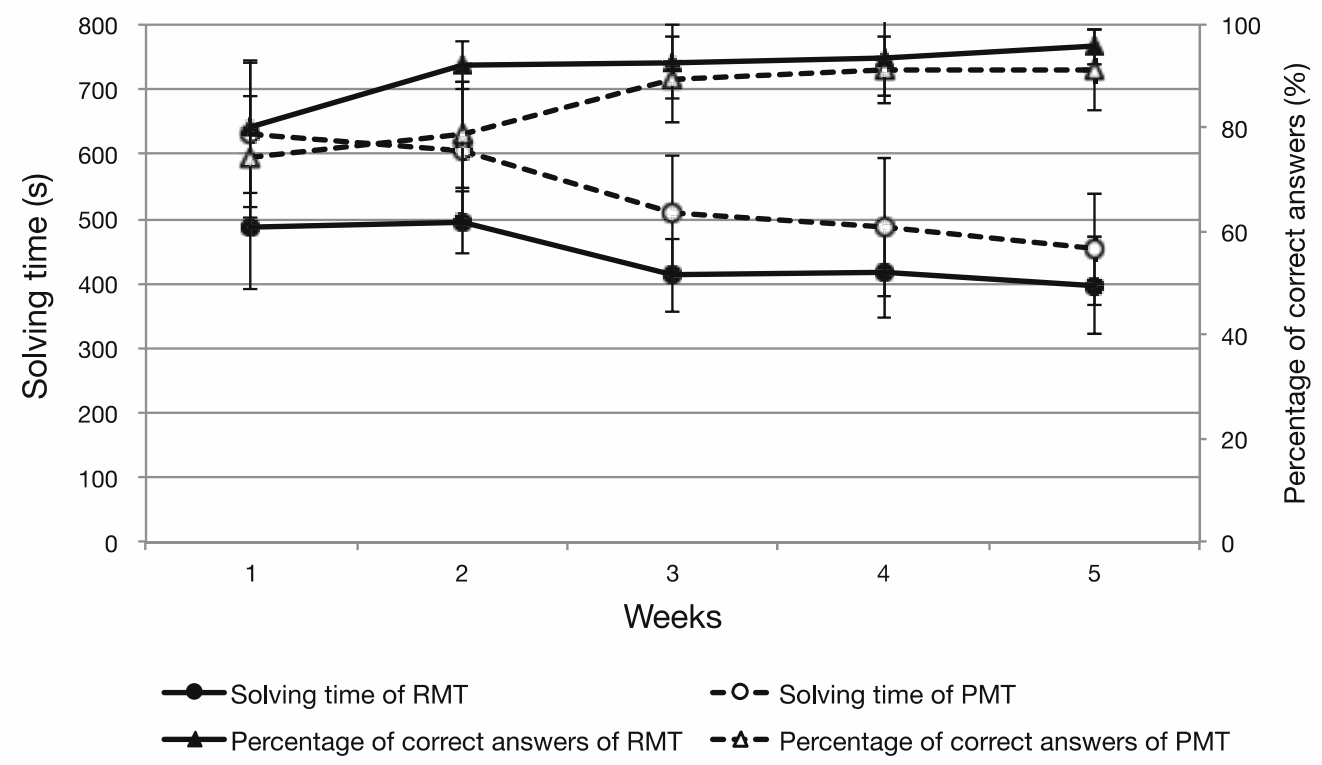

Figure 3. Progression of the solving time and percentage of correct answers. 


\section{Conclusions}

This paper presents our reconstructed version of MathTOUCH using JavaScript to make MathTOUCH available not only on Java-compliant devices but also on various other devices. In this paper, we presented the results of the five-week learning experiment for testing the stability of reconstructed MathTOUCH. The results showed that students are able to study using reconstructed MathTOUCH on STACK as well as the previous version of MathTOUCH. We have made a web trial version of MathTOUCH available to everyone (Fukui, 2016).

The most important avenues for future research are to make the conversion prediction of MathTOUCH intelligent using machine learning to prevent students from having to convert each element individually. In 2015, we proposed a predictive algorithm for converting linear strings to an entire mathematical expression (Shirai and Fukui, 2016). In the future, we plan to implement this algorithm to enhance MathTOUCH. Furthermore, we additionally aim to develop a MathTOUCH interface for smart devices.

\section{Acknowledgements}

This work was supported by JSPS KAKENHI Grant Number JP26330413 and 16K16178.

\section{References}

Osaka Prefecture University, 2016. MATH ON WEB. Available at: http://www.las.osakafuu.ac.jp/lecture/math/MathOnWeb/ [Accessed 8 October. 2016].

Pollanen, M., Wisniewski, T., Yu, X., 2007. Xpress: a novice interface for the real-time communication of mathematical expressions. Proceedings of MathUI 2007. Available at: http://euclid.trentu.ca/math/marco/papers/3 Pollanen-Xpress.pdf [Accessed 5 October 2016].

Smithies, S., Novins, K., Arvo, J., 2001. Equation Entry and Editing via Handwriting and Gesture Recognition. Behaviour and Information Technology, 20(1), pp.53-67.

Fukui, T., 2012. An Intelligent Method of Interactive User Interface for Digitalized Mathematical Expressions (in Japanese). RIMS Kokyuroku, 1780, pp.160-171.

Shirai, S. and Fukui, T., 2014. Improvement in the Input of Mathematical Formulae into STACK using Interactive Methodology (in Japanese). Computer \& Education, 37, pp.85-90.

Shirai, S., Nakamura, Y., and Fukui, T., 2015. An Interactive Math Input Method for Computer Aided Assessment Systems in Mathematics (in Japanese). IPSJ Transactions on Computers and Education, 1(3), pp.11-21.

Shirai, S. and Fukui, T., 2016. Evaluation of a Predictive Algorithm for Converting Linear Strings to Mathematical Formulae for an Input Method. Mathematical Aspects of Computer and Information Sciences, Lecture Notes in Computer Science, 9582, pp.421-425.

Fukui, T., 2016. MathTOUCH. Available at: http://math.mukogawa-u.ac.jp/en/ [Accessed 9 November 2016]. 